\title{
3D Printed Antenna-on-Package with Near-isotropic Radiation Pattern for IoT (WiFi Based) Applications
}

\author{
Zhen Su, Kirill Klionovski, Rana Mohammad Bilal, Atif Shamim \\ Integrated Microwave Packaging Antennas and Circuits Technology Laboratory \\ King Abdullah University of Science and Technology (KAUST) \\ Thuwal, Kingdom of Saudi Arabia \\ su.zhen@kaust.edu.sa, kirill.klionovski@kaust.edu.sa, ranamuhammad.bilal@kaust.edu.sa, atif.shamim@kaust.edu.sa
}

\begin{abstract}
Futuristic IoT applications demand antenna designs to fulfill a number of challenging performance metrics. The antennas for such applications must be small to be easily integrate-able with miniaturized devices, have near-isotropic radiation pattern to be able to communicate in an orientation insensitive way, provide sufficient bandwidth $(4 \%$ for $2.4 \mathrm{GHz}$ WiFi) requisite of concerned communication protocol and be low cost enough to be implemented in billions of devices. Also, as these antennas integrate with the devices in very small spaces, so the effect of components (such as battery, chips etc.) also require consideration in design. This paper presents for the first time, a WiFi $2.4 \mathrm{GHz}$ near-isotropic T-shaped monopole antenna additively manufactured on the package. The antenna is designed whilst taking battery and WiFi chip effect into consideration. The maximum gain is $1.78 \mathrm{dBi}$ with a gain deviation of $6.84 \mathrm{~dB}$. The VSRW=2 bandwidth is achieved from 2.4 to $2.7 \mathrm{GHz}$.
\end{abstract}

Keywords-T-shaped monopole antenna; near-isotropic radiation pattern; additive manufactured antenna.

\section{INTRODUCTION}

Internet of Things (IoT) is an exciting paradigm, where a large number of small devices of everyday use, such as dishwasher, cabinets, coffee maker etc., are connected together to deliver smart services. A significant portion of these devices uses WiFi for the connectivity, as it is predominantly available in most indoor locations. The requisite antenna design needed by these devices is quite challenging, as it must be small in size for easy integration with miniaturized IoT devices, provide near-isotropic radiation pattern for orientation insensitive communication capability, be able to cover complete requisite WiFi band from 2.4 to $2.5 \mathrm{GHz}$, and be low cost enough for implementation on billions of devices.

Certain antenna designs that provide near-isotropic radiation patterns, and thus are suitable for orientation insensitive communication, have been previously reported in the literature [1-7]. Table 1 provides a brief comparison of these designs. As can be seen, the designs from [1, 3, 4] provide sufficiently wide bandwidth $(\mathrm{BW}>4 \%)$. These are, however, 2D antennas with large electrical lengths. 2D Antennas are inherently in-advantageous as compared to 3D Antenna designs, as they take up a large planer space and lose communication completely if antenna face is placed on the ground. Also, the designs from $[1,3,4]$ do not benefit from the antenna on package (AoP) concept or additive manufacturing for cost reduction and miniaturization $[8,9]$. Papers $[2,5]$ have reported additively manufactured 3D wire AoPs with nearisotropic radiation patterns. These antennas, however, have narrow (less than 2\%) bandwidth. Papers [6, 7] have presented electrically small antennas with 3D antenna geometry and near-isotropic radiation patterns. However, these antennas are neither additively manufactured nor AoP. Also, the achieved BW is very narrow (less than $2 \%$ ). Summarizing, an additively manufactured 3D AoP with near-isotropic radiation pattern with sufficiently wide BW has not been reported in the literature before.

In this paper, we present an additively manufactured $\mathrm{T}$ shaped monopole 3D AoP. The presented antenna design takes effect of WiFi chip and battery present inside the IoT device package into consideration. The antenna has a near-isotropic radiation pattern at the frequency of $2.4 \mathrm{GHz}$ and provides a bandwidth of $11.7 \%$. The increased bandwidth ensures that antenna remains properly matched at requisite frequencies, even if the working band slightly shifts because of proximity to a human body or some other external element.

Table 1: Comparison of antennas with near-isotropic radiation patterns

\begin{tabular}{|c|c|c|c|c|c|}
\hline Paper & Geometry & Length & BW & $\begin{array}{c}\text { Additive } \\
\text { manufacturing }\end{array}$ & AoP \\
\hline$[1]$ & $2 \mathrm{D}$ & $>\lambda / 2$ & $8.5 \%$ & No & No \\
\hline$[2]$ & $3 \mathrm{D}$ & $\lambda / 2$ & $1.3 \%$ & Yes & Yes \\
\hline$[3]$ & $2 \mathrm{D}$ & $>\lambda$ & $11 \%$ & No & No \\
\hline$[4]$ & $2 \mathrm{D}$ & $>\lambda$ & $20.82 \%$ & No & No \\
\hline$[5]$ & $3 \mathrm{D}$ & $1.5 \lambda$ & $\mathrm{N} / \mathrm{A}$ & Yes & Yes \\
\hline$[6]$ & $3 \mathrm{D}$ & $\lambda / 4$ & $1.125 \%$ & No & No \\
\hline$[7]$ & $3 \mathrm{D}$ & $0.4 \lambda$ & $1.8 \%$ & No & No \\
\hline $\begin{array}{c}\text { This } \\
\text { work }\end{array}$ & 3D & $0.5 \lambda$ & $11.7 \%$ & Yes & Yes \\
\hline
\end{tabular}




\section{The Antenna Design And Results}

\section{A. The Design of The Antenna on Package}

A T-shaped monopole AoP has been designed in Ansoft HFSS software with a battery and a WiFi chip inside the package (Fig. 1 (a)). The package has the dimensions (in $\mathrm{mm}$ ):
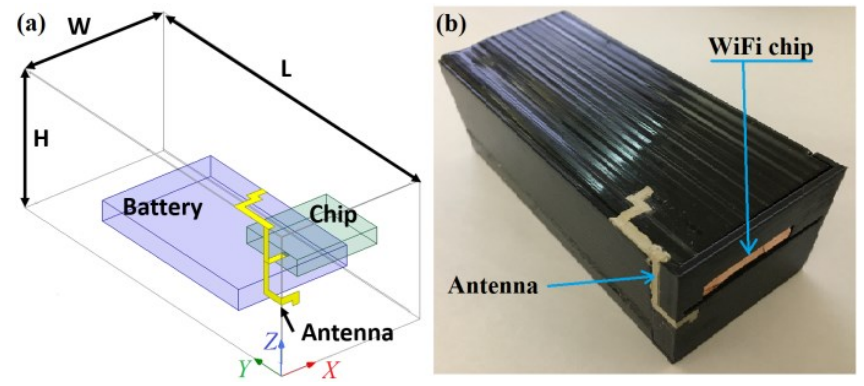

Fig. 1. (a) the AoP design; (b) photo of the additively manufactured AoP.

$\mathrm{L}=106.8, \mathrm{~W}=43.6, \mathrm{H}=40$. The battery is a standard BL-5C LiIon battery (the dimensions of the battery are: $54 \times 34 \times 6 \mathrm{~mm}$ ), and the WiFi chip is RN171 WiFi SOC from Microchip (the dimensions of the chip are: $25 \times 20 \times 4.8 \mathrm{~mm}$ ). Since both these components have metallic shields, they are simulated as perfect electric conductors in the design. The antenna consists of two arms: the upward and the downward arm (along Z-axis, relative to WiFi chip). The upward arm of the antenna has a length of $31.2 \mathrm{~mm}$, and downward arm has a length of 25.6 $\mathrm{mm}$. The WiFi chip acts as a ground for the antenna. The radiation pattern of the $\mathrm{T}$-shaped monopole is formed by the segments that are located along $\mathrm{Z}$ - and $\mathrm{Y}$-axes. The antenna is bent along the $\mathrm{X}$-axis for matching.

\section{B. The Additively Manufactured Prototype}

The AoP was fabricated using Stratasys Objet260 Connex 1 3D printer (Fig. 1 (b)). The printer uses propriety 'Vero Black Plus' material (the permittivity is 2.8 , and the loss tangent is 0.02 at the frequency of $1 \mathrm{GHz}$ ), and provides 30 um minimum vertical resolution. The T-shaped monopole antenna was screen printed on the 3D printed package using propriety silver ink from DuPont with resistivity smaller than $10 \mathrm{~m} \Omega / \mathrm{sq} / 25 \mathrm{um}$.

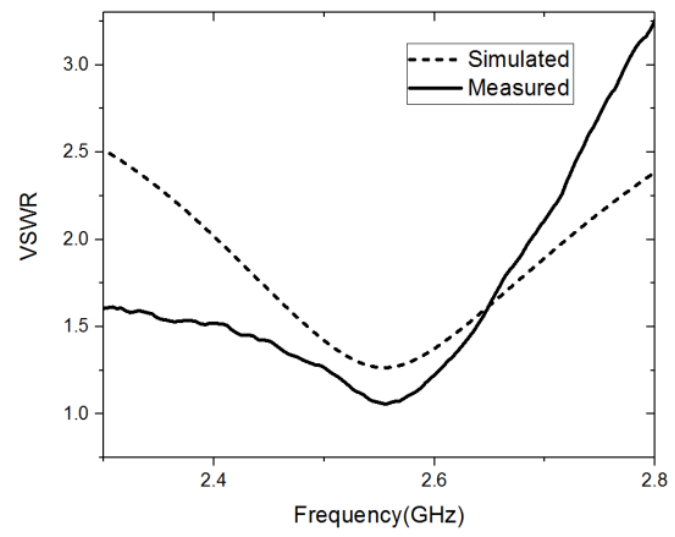

Fig. 2. VSWR of the T-shaped monopole AoP.

\section{The Simulated and Measured Results}

Fig. 2 shows simulated and measured voltage standing wave ratio (VSWR) of the T-shaped monopole AoP. The simulated bandwidth of $11.7 \%$ for the level of VSWR less than 2 is achieved for the frequencies of 2.4-2.72 GHz. Fig. 3 shows the simulated radiation pattern of the AoP at the frequency of

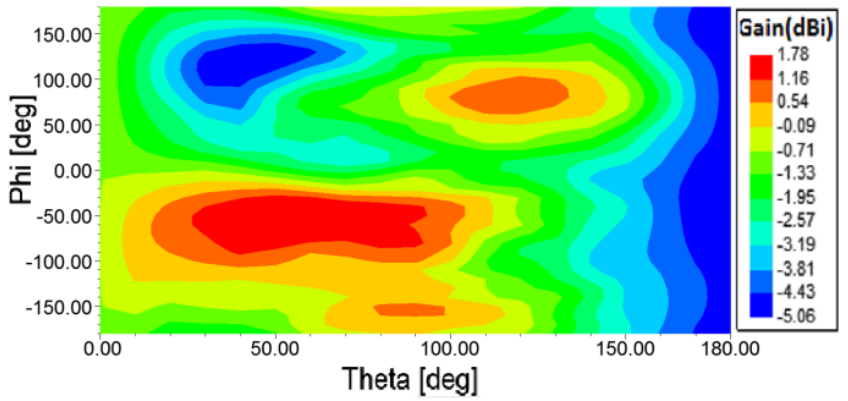

Fig. 3. Simulated radiation pattern of the AoP at the frequency of $2.45 \mathrm{GHz}$.

$2.45 \mathrm{GHz}$. A maximum gain of $1.78 \mathrm{dBi}$ with a minimum gain of $-5.06 \mathrm{dBi}$ is achieved (the gain deviation is $6.84 \mathrm{~dB}$ ).

\section{CONCLUSIONS}

A T-shaped monopole antenna on the package, which provides a near isotropic radiation pattern with a simulated gain deviation of $6.84 \mathrm{~dB}$ and a maximum gain of $1.78 \mathrm{dBi}$ inside the frequency band of $2.4-2.72 \mathrm{GHz}$, is presented. A prototype of the antenna on the package was fabricated using $3 \mathrm{D}$ and screen printing techniques.

\section{REFERENCES}

[1] C. Cho, H. Choo, and I. Park, "Broadband RFID tag antenna with quasiisotropic radiation pattern," Electronics Letters, vol. 41, no. 20, pp. 1091-1092, Sept. 2005.

[2] C. M. Kruesi, R. J. Vyas, and M. M. Tentzeris, "Design and development of a novel 3-D cubic antenna for wireless sensor networks (WSNs) and RFID applications," IEEE Trans. Antennas Propag., vol. 57, no. 10, pp. 3293-3299, Oct. 2009.

[3] G. Pan, Y. Li, Z. Zhang, and Z. Feng, "Isotropic Radiation From a Compact Planar Antenna Using Two Crossed Dipoles," IEEE Antennas Wireless Propag. Lett., vol. 11, pp. 1338-1341, 2012.

[4] C. Deng, Y. Li, Z. Zhang, and Z. Feng, "A Wideband Isotropic Radiated Planar Antenna Using Sequential Rotated L-Shaped Monopoles," IEEE Trans. Antennas Propag., vol. 62, no. 3, pp. 1461-1464, March 2014.

[5] M. F. Farooqui, C. Claudel, and A. Shamim, "An Inkjet-Printed Buoyant 3-D Lagrangian Sensor for Real-Time Flood Monitoring," IEEE Trans. Antennas Propag., vol. 62, no. 6 pp. 3354-3359, June 2014.

[6] C. Deng, Y. Li, Z. Zhang, and Z. Feng, "Design Of a ThreeDimensional Folded Slot Antenna With Quasi-Isotropic Radiation Pattern," in Proc. Antennas Propag. Soc. Int. Symp., Vancouver, BC, Canada, July 2015, pp. 588-589.

[7] J. H. Kim and S. Nam, "A Compact Quasi-Isotropic Antenna Based on Folded Split Ring Resonators," IEEE Antennas Wireless Propag. Lett., vol. 16, pp. 294-297, 2017.

[8] M. F. Farooqui, M. A.Karimi, K. N. Salama, A. Shamim, "3D-Printed Disposable Wireless Sensors with Integrated Microelectronics for Large Area Environmental Monitoring", J. Advanced Materials Technologies, vol. 2, no. 8, 2017.

[9] Z. Su, A. Shamim, "A 3D printed dual GSM band near isotropic onpackage antenna," in Proc. Antennas Propag. Soc. Int. Symp., San Diego, CA, USA, July 2017, pp. 1251-1252. 